\title{
Invariant Poisson Realizations and the Averaging of Dirac Structures
}

\author{
José A. VALLEJO ${ }^{\dagger}$ and Yurii VOROBIEV ${ }^{\ddagger}$ \\ † Facultad de Ciencias, Universidad Autónoma de San Luis Potosí, México \\ E-mail: jvallejo@fc.uaslp.mx \\ URL: http://galia.fc.uaslp.mx/ jvallejo/ \\ ‡Departamento de Matemáticas, Universidad de Sonora, México \\ E-mail: yurimv@guaymas.uson.mx
}

Received May 19, 2014, in final form September 09, 2014; Published online September 15, 2014

http://dx.doi.org/10.3842/SIGMA.2014.096

\begin{abstract}
We describe an averaging procedure on a Dirac manifold, with respect to a class of compatible actions of a compact Lie group. Some averaging theorems on the existence of invariant realizations of Poisson structures around (singular) symplectic leaves are derived. We show that the construction of coupling Dirac structures (invariant with respect to locally Hamiltonian group actions) on a Poisson foliation is related with a special class of exact gauge transformations.
\end{abstract}

Key words: Poisson structures; Dirac structures; geometric data; averaging operators

2010 Mathematics Subject Classification: 53D17; 70G45; 53C12

\section{Introduction}

Our aim is to discuss some aspects of the averaging procedure on Poisson manifolds which carry singular symplectic foliations.

Let $(M, \Pi)$ be a Poisson manifold endowed with a Poisson tensor $\Pi$. The characteristic distribution generated by the Hamiltonian vector fields on $(M, \Pi)$ is integrable in the sense of Stefan-Sussmann $[20,21]$, and gives rise to the smooth symplectic foliation $(\mathcal{S}, \omega)$, having a leafwise symplectic form $\omega$. The singular situation occurs when there are points where the rank of the Poisson tensor $\Pi$ is not locally constant. In this case the leaf-wise symplectic form $\omega$ has a singular behavior, in the sense that $\omega$ can not be represented as the pull-back of a global 2form on $M$. Given a leaf-preserving (non-canonical) action of a compact connected Lie group $G$ on $M$, we are interested in the existence of an invariant realization of $\Pi$ around a (singular) symplectic leaf $S$, that is, a $G$-invariant Poisson structure $\bar{\Pi}$ which is Poisson-isomorphic to $\Pi$ on a neighborhood of $S$. Such invariant Poisson realizations appear naturally in the theory of normal forms for Hamiltonian systems of adiabatic type on general phase spaces [1,28], which is a motivation for the present work.

Our intention is to describe a natural reconstruction procedure for a $G$-invariant Poisson structure $\bar{\Pi}$ from the original one $\Pi$. It is clear that, in the contravariant setting, the standard averaging technique [16] does not work because of the nonlinear character of the Jacobi identity. The alternative proposed here is the construction of an invariant Poisson structure $\bar{\Pi}$ by applying averaging arguments to the leaf-wise symplectic form $\omega$. In doing so, we must deal with smoothness and non-degeneracy issues, which are not trivial at all in the singular case. The crucial point is that the smoothness condition for leaf-wise pre-symplectic forms can be formulated within the category of Dirac structures $[7,8]$. This allows us to develop the reconstruction procedure within the framework of the Dirac formalism, starting with the Dirac structure $D=$ Graph $\Pi \subset T M \oplus$ $T^{*} M$ associated with $\Pi$. We remark that if the $G$-action is compatible in an appropriate way 
with the leaf-wise pre-symplectic form $\omega$, then its $G$-average is a smooth leaf-wise pre-symplectic form on $\mathcal{S}$ inducing a $G$-invariant Dirac structure $\bar{D}$. Moreover, the Dirac structures $\bar{D}$ and $D$ are related by a gauge transformation $[4,5,18]$ associated to an exact 2 -form on $M$. Therefore, by the averaging procedure we mean here the passage from $D$ to $\bar{D}$. When a non-degeneracy condition holds, the Dirac structure $\bar{D}$ is the graph of a Poisson bi-vector field $\bar{\Pi}$ with the property that $\bar{\Pi}$ is invariant with respect to the $G$-action, and gauge-equivalent to $\Pi$. Combining these arguments with the Moser path method for Poisson structures [9, 12, 17, 26], we get that the Poisson structure $\bar{\Pi}$ gives an invariant realization of $\Pi$ around the symplectic leaf $S$.

If the manifold $M$ carries the additional structure of a regular foliation $\mathcal{F}$, we apply the above results to the class of $\mathcal{F}$-coupling Poisson tensors [23, 25]. Let $\Pi=\Pi_{2,0}+\Pi_{0,2}$ be a coupling Poisson tensor on $(M, \mathcal{F})$, where the "regular part" $\Pi_{2,0} \in \Gamma\left(\wedge^{2} \mathbb{H}\right)$ is a bi-vector field of constant rank, and the "singular part" $\Pi_{0,2} \in \Gamma\left(\wedge^{2} \mathbb{V}\right)$ is a leaf-tangent Poisson tensor. We show that, if the $G$-action is compatible with the singular part $\Pi_{0,2}$ and the symplectic leaf $\mathcal{S}$ of $\Pi$ is transversal to the foliation $\mathcal{F}$, then $\Pi$ admits an invariant realization around the leaf which is again a $\mathcal{F}$-coupling Poisson structure $\bar{\Pi}=\bar{\Pi}_{2,0}+\bar{\Pi}_{0,2}$, with $G$-invariant regular and singular parts. In particular, the compatibility condition automatically holds when the $G$-action is locally Hamiltonian on $\left(M, \Pi_{0,2}\right)$.

We also present an alternative approach to the construction of Dirac manifolds with symmetry within the class of coupling Dirac structures [2, 10, 11, 24, 29]. Starting with a Poisson foliation $(M, \mathcal{F}, P)$ endowed with the locally Hamiltonian action of a compact Lie group $G$, we describe an averaging procedure $D \mapsto \bar{D}$, for compatible $\mathcal{F}$-coupling Dirac structures $D$ on $(M, \mathcal{F}, P)$, in terms of the gauge transformations of the corresponding integrable geometric data [27]. This approach is based on the averaging technique for Poisson connections originally developed, for Hamiltonian group actions on Poisson fiber bundles, in [16]. Here we use a foliated version of this technique which does not require the existence of a global momentum map. With a different perspective, an averaging procedure was also introduced in $[13,14]$, to construct induced Dirac structures in the context of the reduction method on Dirac manifolds with symmetry.

The paper is organized as follows: General properties of averaging operators are reviewed in Section 2. In Section 3, we describe the averaging procedure on Dirac manifolds with respect to a class of compatible $G$-actions, and study its relation with exact gauge transformations. In Section 4, we formulate and prove the Poisson averaging theorem on the existence of invariant realizations of Poisson structures around (singular) symplectic leaves. In Section 5, within the class of coupling Poisson structures on a foliated manifold, the $G$-invariant splittings for Poisson models around a symplectic leaf transversal to the foliation, are described by using the bi-graded contravariant calculus and gauge type transformations. Section 6 is devoted to the study of some symmetries of the structure equations (integrability conditions) for geometric data on Poisson foliations. We describe a class of gauge transformations of integrable geometric data which are equivalent with exact gauge transformations of Dirac structures preserving the coupling property. In Section 7, these results are used for the construction of coupling Dirac structures on a Poisson foliation, invariant with respect to locally Hamiltonian $G$-action, in terms of the "averaged" integrable geometric data. We also describe some cohomological obstructions to the construction of Dirac manifolds with Hamiltonian $G$-symmetry in the context of the averaging procedure.

\section{Averaging operators}

Let $G$ be a compact connected Lie group and $\mathfrak{g}$ its Lie algebra. Suppose we are given a smooth (left) action $\Phi: G \times M \rightarrow M,(g, m) \mapsto \Phi(g, m)=\Phi_{g}(m)$. Denote by $a_{M} \in \chi(M)$ the infinitesimal generator of $\Phi$ associated to an element $a \in \mathfrak{g}$,

$$
a_{M}(m)=\left.\frac{\mathrm{d}}{\mathrm{d} t}\right|_{t=0} \Phi_{\exp (t a)}(m) .
$$


Let $\mathcal{T}_{s}^{r}(M)$ be the space of all smooth tensor fields on $M$ of type $(r, s)$. The $G$-average of every $F \in \mathcal{T}_{s}^{r}(M)$ is a tensor field of the same type $\langle F\rangle^{G} \in \mathcal{T}_{s}^{r}(M)$ given by the formula

$$
\langle F\rangle^{G}:=\int_{G} \Phi_{g}^{*} F \mathrm{~d} g,
$$

where $\mathrm{d} g$ is the normalized Haar measure on $G$. A tensor field $F$ is said to be $G$-invariant if $\Phi_{g}^{*} F=F$ for any $g \in G$ or, equivalently, $\langle F\rangle^{G}=F$. In infinitesimal terms, using the Lie derivative, the $G$-invariance of $F \operatorname{reads} \mathcal{L}_{a_{M}} F=0 \forall a \in \mathfrak{g}$. Moreover, we have the useful identity $\left\langle\mathcal{L}_{a_{M}} F\right\rangle^{G}=0$, for every $F \in \mathcal{T}_{s}^{r}(M)$.

Since $G$ is compact and connected, the exponential mappings exp : $\mathfrak{g} \rightarrow G$, constructed from the Lie group structure and from the corresponding bi-invariant Riemannian structure, coincide, and this map is surjective. Consider the cut locus $C$ of the identity $e \in G$. Then,

$$
\left.\exp \right|_{\mathcal{D}}: \mathcal{D} \rightarrow G \backslash C
$$

is a diffeomorphism between an open, bounded, star-shaped neighborhood $\mathcal{D}$ of $0 \in \mathfrak{g}$, and the complement $G \backslash C$. Moreover, $\exp (\partial \mathcal{D})=C$ has zero measure (these are standard results in Riemannian geometry; for instance, see chapter III in [6]). Let $\mu=\mathrm{d} g$ be the normalized Haar measure on $G$, considered as a left (right) volume form on $G$, and denote by $\operatorname{Hom}\left(\mathfrak{g} ; \mathcal{T}_{s}^{r}(M)\right)$ the space of $\mathbb{R}$-linear mappings $\lambda: \mathfrak{g} \rightarrow \mathcal{T}_{s}^{r}(M)$. Then, we can define an averaging operator $\delta^{G}: \operatorname{Hom}\left(\mathfrak{g} ; \mathcal{T}_{s}^{r}(M)\right) \rightarrow \mathcal{T}_{s}^{r}(M)$ as follows:

$$
\delta^{G}(\lambda):=\int_{\mathcal{D}}\left(\int_{0}^{1} \Phi_{\exp (t a)}^{*} \lambda_{a} \mathrm{~d} t\right) \exp ^{*} \mu .
$$

Example 2.1. Let $G=\mathbb{S}^{1}=\mathbb{R} \backslash 2 \pi \mathbb{Z}$. Suppose that an $\mathbb{S}^{1}$-action is generated by the $2 \pi$-periodic flow of a vector field $\Upsilon$ on $M$. Then, formula (2.2) reads

$$
\delta^{G}(\lambda)=-\frac{1}{2 \pi} \int_{0}^{2 \pi}(t-\pi)\left(\mathrm{Fl}_{\Upsilon}^{t}\right)^{*} F \mathrm{~d} t+\pi\langle F\rangle^{\mathbb{S}^{1}},
$$

where $\lambda=a F, F \in \mathcal{T}_{s}^{r}(M)$, and $a \in \mathbb{R}$.

Consider also the mapping $l^{G}: \mathcal{T}_{s}^{r}(M) \rightarrow \operatorname{Hom}\left(\mathfrak{g} ; \mathcal{T}_{s}^{r}(M)\right)$ given by

$$
l^{G}(F): \mathfrak{g} \ni a \mapsto \mathcal{L}_{a_{M}} F .
$$

The following useful fact follows from standard averaging arguments [16].

Lemma 2.2. The averaging operator $\langle\cdot\rangle^{G}: \mathcal{T}_{s}^{r}(M) \rightarrow \mathcal{T}_{s}^{r}(M)$ has the representation:

$$
\langle\cdot\rangle^{G}=\mathrm{id}+\delta^{G} \circ l^{G} .
$$

Proof. Firstly, we have (for any $F \in \mathcal{T}_{s}^{r}(M)$ ),

$$
\Phi_{\exp a}^{*} F-F=\int_{0}^{1} \Phi_{\exp t a}^{*}\left(\mathcal{L}_{a_{M}} F\right) \mathrm{d} t .
$$

On the other hand, since $C$ is a subset of measure 0 , the average of every $F \in \mathcal{T}_{s}^{r}(M)$ can be written as

$$
\langle F\rangle^{G}=\int_{\mathcal{D}}\left(\Phi_{\exp a}^{*} F\right) \exp ^{*} \mu .
$$

Using this property, and integrating equation (2.4) over $\mathcal{D}$, we get (2.3).

The operators $\langle\cdot\rangle^{G}, \delta^{G}$, and $l^{G}$ can be restricted to the spaces of multi-vector fields $\chi^{k}(M)$ and differential forms $\Omega^{k}(M)$. In particular, it follows from (2.3) that the $G$-average of a closed $k$-form, $\beta \in \Omega_{\mathrm{cl}}^{k}(M)$, is

$$
\langle\beta\rangle^{G}=\beta-\mathrm{d} \circ \delta^{G}(\rho),
$$

where $\rho \in \operatorname{Hom}\left(\mathfrak{g} ; \Omega^{k}(M)\right)$ is given by the insertion operator $\rho_{a}:=-\mathbf{i}_{a_{M}} \beta$. 


\section{Compatible group actions}

\subsection{Generalities on Dirac structures}

First, we recall some basic properties of Dirac structures which can be found, for example, in $[4,5,7,8,13]$.

A Dirac structure on a manifold $M$ is a smooth distribution $D \subset T M \oplus T^{*} M$ which is maximally isotropic with respect to the natural symmetric pairing

$$
\langle(X, \alpha),(Y, \beta)\rangle:=\beta(X)+\alpha(Y),
$$

and involutive with respect to the Courant bracket

$$
[(X, \alpha),(Y, \beta)]:=\left([X, Y], \mathcal{L}_{X} \beta-\mathcal{L}_{Y} \alpha+\frac{1}{2} \mathrm{~d}(\alpha(Y)-\beta(X))\right) .
$$

Here $(X, \alpha) \in \Gamma(D)$ is a (local) smooth section of $D$. Let $p_{T}: T M \oplus T^{*} M \rightarrow T M$ be the natural projection. It follows that $\operatorname{rank} D=\operatorname{dim} M$, and the characteristic distribution $\mathcal{C}=p_{T}(D) \subset T M$ is integrable in the sense of Stefan and Sussmann. As a consequence, the Dirac manifold $(M, D)$ carries a (singular) pre-symplectic foliation $(\mathcal{S}, \omega)$ : the leaves are maximal integral manifolds of $\mathcal{C}=T \mathcal{S}$, and the leaf-wise pre-symplectic structure $\omega$ is defined by $\omega_{m}(X, Y)=-\alpha(Y)$, where $(X, Y) \in \mathcal{C}_{m}$ and $(X, \alpha) \in D_{m}$. In particular, the foliation $(\mathcal{S}, \omega)$ is symplectic if and only if $D$ is the graph of a Poisson structure on $M$. Reciprocally, one can associate to a pre-symplectic foliation $(\mathcal{S}, \omega)$ on $M$, the distribution

$$
D^{\omega}:=\left\{(X, \alpha) \in T_{m} M \oplus T_{m}^{*} M\left|X \in T_{m} \mathcal{S}, \alpha\right|_{T_{m} \mathcal{S}}=-\mathbf{i}_{X} \omega_{m}\right\}
$$

We say that $\omega$ is smooth if $D^{\omega}$ is a smooth sub-bundle of $T M \oplus T^{*} M$. In this case, $D^{\omega}$ is a Dirac structure whose pre-symplectic foliation is just $(\mathcal{S}, \omega)$. Thus, there is a one-to-one correspondence between Dirac structures and smooth pre-symplectic foliations on $M$.

Notice that every Poisson structure on $M$ induces a Dirac structure for which the involutive property follows from the Jacobi identity. Indeed, given a bi-vector field $\Pi \in \Gamma\left(\wedge^{2} T M\right)$, we define the smooth sub-bundle

$$
D_{\Pi}:=\operatorname{Graph}(\Pi)=\left\{(X, \alpha) \in T_{m} M \oplus T_{m}^{*} M \mid X=\mathbf{i}_{\alpha} \Pi\right\} .
$$

Then, $D_{\Pi}$ is a Dirac structure if and only if $\llbracket \Pi, \Pi \rrbracket=0$, that is, $\Pi$ is a Poisson bi-vector field. Here $\llbracket \cdot, \cdot \rrbracket$ denotes the Schouten bracket for multi-vector fields on $M[22]$. In this case, $(\mathcal{S}, \omega)$ is in fact the symplectic foliation of the Poisson structure $\Pi$. Reciprocally, if the characteristic foliation of a Dirac structure $D$ is symplectic, then $D$ is the graph of a Poisson structure.

One can modify the leaf-wise pre-symplectic structure $\omega$ of a Dirac structure $D$ by using the pull back of a closed 2-form $B \in \Omega^{2}(M)$ : for each pre-symplectic leaf $\left(S, \omega_{S}\right)$, we define the new pre-symplectic structure as $\omega_{S}-\iota_{S}^{*} B$, where $\iota_{S}: S \hookrightarrow M$ is the inclusion map. Then, the foliation $\mathcal{S}$ endowed with the deformed leaf-wise pre-symplectic structure gives rise to the new Dirac structure $\tau_{B}(D)=\left\{\left(X, \alpha-\mathbf{i}_{X} B\right):(X, \alpha) \in D\right\}$. Therefore, for every closed 2-form $B$, the transformation $\tau_{B}$ (called the gauge transformation $[5,18]$ ) sends Dirac structures to Dirac structures.

A Dirac structure $D$ on $M$ is said to be invariant with respect to a diffeomorphism $\phi$ : $M \rightarrow M$ if $\left(\phi^{*} X, \phi^{*} \alpha\right) \in \Gamma(D)$ for every $(X, \alpha) \in \Gamma(D)$. In this case, $\phi$ is called a Dirac diffeomorphism. In particular, if $D=$ Graph $\Pi$ is the Dirac structure associated to a Poisson bi-vector field $\Pi$ on $M$, then the $\phi$-invariance of $D$ is equivalent to the condition $\phi^{*} \Pi=\Pi$, that is, $\phi$ is a Poisson diffeomorphism. An action of a Lie group on $(M, D)$ by Dirac diffeomorphisms is called canonical. 
A vector field $X$ on $M$ is Hamiltonian relative to a Dirac structure $D$ if there exists a function $F \in C^{\infty}(M)$ such that

$$
(X, \mathrm{~d} F) \in \Gamma(D) .
$$

A $G$-action on $(M, D)$ by Dirac diffeomorphisms is said to be Hamiltonian, with momentum map $J \in \operatorname{Hom}\left(\mathfrak{g} ; C^{\infty}(M)\right)$, if the infinitesimal generator $a_{M}$ of every $a \in \mathfrak{g}$ is a Hamiltonian vector field,

$$
\left(a_{M}, \mathrm{~d} J_{a}\right) \in \Gamma(D) .
$$

The integrability and reduction for these actions has been studied in [3].

\subsection{Averaging procedure: $D \mapsto \bar{D}$}

Now, let $(M, D)$ be a Dirac manifold and $(\mathcal{S}, \omega)$ the associated pre-symplectic foliation. Suppose we are given the action of a connected compact Lie group $G$ on $M$ which preserves each leaf of $\mathcal{S}$ but is not necessarily canonical relative to $D$. Therefore, the $G$-action is tangent to the presymplectic leaves, $a_{M}(m) \in T_{m} \mathcal{S}$, for all $m \in M, a \in \mathfrak{g}$. Applying the averaging operator (2.1) to $\omega_{S}$ on every pre-symplectic leaf $\left(S, \omega_{S}\right)$, gives the averaged leaf-wise pre-symplectic form $\langle\omega\rangle^{G}$ on $\mathcal{S}$.

We say that the leaf-preserving $G$-action on the Dirac manifold $(M, D)$ is compatible if there exists a $\mathbb{R}$-linear mapping $\rho \in \operatorname{Hom}\left(\mathfrak{g}, \Omega^{1}(M)\right)$ such that, for each leaf $S$,

$$
\mathbf{i}_{a_{M}} \omega_{S}=-i_{S}^{*} \rho_{a},
$$

for every $a \in \mathfrak{g}$, where $i_{S}: S \hookrightarrow M$ is the canonical injection. This compatibility condition can be rewritten as follows

$$
\left(a_{M}, \rho_{a}\right) \in \Gamma(D), \quad \forall a \in \mathfrak{g} .
$$

It is clear that this condition always holds for Hamiltonian $G$-actions on $(M, D)$, and also when the pre-symplectic foliation is regular (of constant rank).

Proposition 3.1. If the $G$-action is compatible on $(M, D)$, then the average $\langle\omega\rangle^{G}$ is smooth, and can be represented as

$$
\langle\omega\rangle^{G}=\omega-i_{S}^{*} \mathrm{~d} \Theta
$$

where $\Theta \in \Omega^{1}(M)$ is the 1-form given by

$$
\Theta:=\delta^{G}(\rho) .
$$

The associated Dirac structure $\bar{D}:=D^{\langle\omega\rangle^{G}}$ is $G$-invariant and related to $D$ by an exact gauge transformation,

$$
\bar{D}=\left\{\left(X, \alpha+\mathbf{i}_{X} \mathrm{~d} \Theta\right):(X, \alpha) \in D\right\} .
$$

Proof. It follows directly from (2.5), (3.2) and the properties of the gauge transformations.

Remark 3.2. The 1-form $\Theta$ in (3.3) is defined up to the addition of an arbitrary 1-form on $M$ which is closed on each leaf $S$. It follows from (3.3), and the fact that $\langle\cdot\rangle^{G}$ commutes with pull-backs, that $\iota_{S}^{*}\langle\mathrm{~d} \Theta\rangle^{G}=0$, and hence one can always choose the gauge 1-form having zero average, by making $\Theta^{0}=\Theta-\langle\Theta\rangle^{G}$. 
We will use the following notations. For an arbitrary bi-vector field $\Pi \in \Gamma\left(\wedge^{2} T M\right)$, and a 2-form $B \in \Omega^{2}(M)$, we denote by $\Pi^{\sharp}: T^{*} M \rightarrow T M$ and $B^{\sharp}: T M \rightarrow T^{*} M$ the vector bundle morphisms given by $\alpha \mapsto \mathbf{i}_{\alpha} \Pi$ and $X \mapsto \mathbf{i}_{X} B$, respectively.

Now, we formulate the following "Poisson version" of Proposition 3.1.

Corollary 3.3. Let $(M, \Pi)$ be a Poisson manifold and $(\mathcal{S}, \omega)$ its symplectic foliation. Suppose that an action of a compact connected Lie group $G$ on $M$ is compatible with the Poisson tensor $\Pi$, in the sense that

$$
a_{M}=\Pi^{\sharp} \rho_{a}, \quad \forall a \in \mathfrak{g},
$$

for a certain $\rho \in \operatorname{Hom}\left(\mathfrak{g}, \Omega^{1}(M)\right)$, and consider the 1-form $\Theta$ in (3.4). If the endomorphism

$$
\mathrm{Id}+(\mathrm{d} \Theta)^{\sharp} \circ \Pi^{\sharp}: T^{*} M \rightarrow T^{*} M \text { is invertible, }
$$

then the $G$-average $\langle\omega\rangle^{G}$ is non-degenerate on each leaf of $\mathcal{S}$, and there exists a unique $G$-invariant Poisson tensor $\bar{\Pi}$ on $M$ whose singular symplectic foliation is just $\left(\mathcal{S},\langle\omega\rangle^{G}\right)$. The Poisson structures $\bar{\Pi}$ and $\Pi$ are related by the exact gauge transformation,

$$
\bar{\Pi}^{\sharp}=\Pi^{\sharp} \circ\left(\mathrm{Id}+(\mathrm{d} \Theta)^{\sharp} \circ \Pi^{\sharp}\right)^{-1} .
$$

Therefore, under the non-degeneracy condition (3.6), one can get a Poisson structure which is invariant with respect to the compatible $G$-action.

\section{The averaging theorem around symplectic leaves}

Here, we apply the results of the previous section to the construction of invariant Poisson models around a (singular) symplectic leaf.

Theorem 4.1. Let $(M, \Pi)$ be a Poisson manifold, and $S$ a symplectic leaf of the foliation induced by $\Pi$. Suppose we are given an action of a compact connected Lie group $G$ on $M$, which is compatible with $\Pi$ (recall (3.5)). If $G$ acts canonically on $S$ (but not necessarily on the other leaves), that is, recalling (3.2)

$$
\iota_{S}^{*} \rho_{a} \text { is closed on } S,
$$

then (3.7) determines a $G$-invariant Poisson bi-vector $\bar{\Pi}$, well-defined in a G-invariant neighborhood $N$ of $S$ in $M$. Moreover, if

$$
S \subset M^{G} \quad \text { (the set of fixed points), }
$$

then, the germs at $S$ of $\bar{\Pi}$ and $\Pi$ are isomorphic by a local Poisson diffeomorphism $\phi: N \rightarrow M$,

$$
\phi^{*} \Pi=\bar{\Pi},\left.\quad \phi\right|_{S}=\mathrm{id} .
$$

Proof. Fix a sub-bundle, $\mathbb{V}_{S} \subset T_{S} M$, which is transverse to the symplectic leaf,

$$
T_{S} M=T S \oplus \mathbb{V}_{S} .
$$

Then,

$$
T_{S}^{*} M=\mathbb{V}_{S}^{0} \oplus(T S)^{0},
$$


and it follows from $\Pi^{\sharp}\left(T_{S}^{*} M\right)=T S$ that

$$
\Pi^{\sharp}\left((T S)^{0}\right)=0, \quad \Pi^{\sharp}\left(\mathbb{V}_{S}^{0}\right)=T S .
$$

Let $\Theta$ be the 1-from on $M$ given by (3.4). Consider the bundle morphism $B^{\sharp}: T M \rightarrow T^{*} M$ induced by the 2 -form $B=-\mathrm{d} \Theta$. By condition (4.1), $\Theta$ is closed on $S$, and hence $\iota_{S}^{*} B=0$ or, equivalently,

$$
B^{\sharp}(T S) \subseteq(T S)^{0} .
$$

From that result, and properties (4.5), we get

$$
B^{\sharp} \circ \Pi^{\sharp}\left(\mathbb{V}_{S}^{0}\right) \subseteq(T S)^{0}, \quad \text { and } \quad B^{\sharp} \circ \Pi^{\sharp}\left((T S)^{0}\right)=0 .
$$

These relations, together with (4.4), mean that, for each $t \in[0,1]$, the restriction of the vector bundle morphism Id $-t B^{\sharp} \circ \Pi^{\sharp}$ to $T_{S}^{*} M$ is invertible, with

$$
\left(\mathrm{Id}-t B^{\sharp} \circ \Pi^{\sharp}\right)^{-1}=\mathrm{Id}+t B^{\sharp} \circ \Pi^{\sharp} \quad \text { on } \quad T_{S}^{*} M .
$$

Then, there exists an open neighborhood $N$, of $S$ in $M$, such that the restriction of Id $-t B^{\sharp} \circ \Pi^{\sharp}$ to $T_{N}^{*} M$ is invertible for all $t \in[0,1]$. Since the Lie group $G$ is compact, one can choose the neighborhood $N$ as being $G$-invariant. Applying to $\Pi$ the gauge transformation determined by $t B$, we get a $t$-dependent family of Poisson tensors $\Pi_{t}$ on $N$, such that:

$$
\Pi_{t}^{\sharp}:=\Pi^{\sharp} \circ\left(\operatorname{Id}-t B^{\sharp} \circ \Pi^{\sharp}\right)^{-1} .
$$

This family joins the original Poison structure, $\Pi$, with the $G$-invariant one $\bar{\Pi}=\Pi_{1}$. Next, one can verify $[9,12,25,26]$ that the time-dependent vector field on $N$ given by

$$
Z_{t}=-\Pi_{t}^{\sharp}(\Theta)=-\Pi^{\sharp} \circ\left(\operatorname{Id}+t(\mathrm{~d} \Theta)^{\sharp} \circ \Pi^{\sharp}\right)^{-1}(\Theta),
$$

satisfies the homotopy equation

$$
\llbracket Z_{t}, \Pi_{t} \rrbracket=-\frac{\mathrm{d} \Pi_{t}}{\mathrm{~d} t} .
$$

Finally, hypothesis (4.2) implies that $\left.\Theta\right|_{T_{S} M} \in(T S)^{0}$, and hence, by (4.6), we get $\left.Z_{t}\right|_{S}=0$. Therefore, shrinking if necessary the neighborhood $N$, we can make the flow $\mathrm{Fl}_{Z_{t}}^{t}$ of $Z_{t}$ well defined on $N$, for all $t \in[0,1]$. The Poisson diffeomorphism in (4.3) is then given by the flow at time $1, \phi=\left.\mathrm{Fl}_{Z_{t}}^{t}\right|_{t=1}$.

\section{$5 \quad$ G-invariant splittings}

According to the coupling procedure [25], in a neighborhood of a closed symplectic leaf, a Poisson structure splits into "regular" and "singular" parts, where the singular part is called a transverse Poisson structure of the leaf. In this section, by using Theorem 4.1, we show that, with respect to a class of transversally compatible $G$-actions, such a splitting can be made $G$-invariant, and compute the invariant regular and singular components in terms of gauge transformations.

Let $\mathcal{F}$ be a regular foliation on a manifold $M$. Denote by $\mathbb{V}:=T \mathcal{F}$ the tangent bundle of $\mathcal{F}$, and by $\mathbb{V}^{0} \subset T^{*} M$ its annihilator. Recall that a Poisson bi-vector field, $\Pi \in \Gamma\left(\wedge^{2} T M\right)$, on the foliated manifold $(M, \mathcal{F})$ is said to be $\mathcal{F}$-coupling [23, 25], if the associated distribution

$$
\mathbb{H}:=\Pi^{\sharp}\left(\mathbb{V}^{0}\right),
$$


is a normal (regular) bundle of $\mathcal{F}$, that is,

$$
T M=\mathbb{H} \oplus \mathbb{V},
$$

and hence

$$
T^{*} M=\mathbb{V}^{0} \oplus \mathbb{H}^{0} .
$$

These splittings define an $\mathbb{H}$-dependent bi-grading of differential forms and multi-vector fields on $M$ :

$$
\Omega^{k}(M)=\bigoplus_{p+q=k} \Omega^{p, q}(M), \quad \Gamma\left(\wedge^{k} T M\right)=\bigoplus_{p+q=k} \chi^{p, q}(M)
$$

where the elements of the sub-spaces $\Omega^{p, q}(M)=\Gamma\left(\wedge^{q} \mathbb{V}^{0} \otimes \wedge^{p} \mathbb{H}^{0}\right)$, and $\chi^{p, q}(M)=\Gamma\left(\wedge^{p} \mathbb{H} \otimes \wedge^{q} \mathbb{V}\right)$, are said to be differential forms and multi-vector fields of bi-degree $(p, q)$, respectively. For any $k$-form $\omega$, and $k$-vector field $A$, the terms of bi-degree $(p, q)$ in the above decompositions will be denoted by $\omega_{p, q}$ and $A_{p, q}$, respectively. Moreover, we will use the following bi-graded decomposition of the exterior differential, d, on $M[22,23]$ :

$$
\mathrm{d}=\mathrm{d}_{1,0}+\mathrm{d}_{2,-1}+\mathrm{d}_{0,1} .
$$

For every $\mathcal{F}$-coupling Poison tensor $\Pi$, the mixed term $\Pi_{1,1}$, of bi-degree $(1,1)$, vanishes and we have the decomposition

$$
\Pi=\Pi_{2,0}+\Pi_{0,2},
$$

where the "regular part", $\Pi_{2,0} \in \Gamma\left(\wedge^{2} \mathbb{H}\right)$ is a bi-vector field of constant rank,

$$
\operatorname{rank} \Pi_{2,0}=\operatorname{dim} \mathbb{H}=\operatorname{codim} \mathcal{F},
$$

and the "singular part", $\Pi_{0,2} \in \Gamma\left(\wedge^{2} \mathbb{V}\right)$, is a leaf-wise tangent Poisson tensor,

$$
\Pi_{0,2}^{\sharp}\left(T^{*} M\right) \subset \mathbb{V}, \quad \text { and } \quad \llbracket \Pi_{0,2}, \Pi_{0,2} \rrbracket=0 .
$$

It follows from (5.2) that the restriction of $\Pi_{2,0}^{\sharp}$ to $\mathbb{V}^{0}$ is a vector bundle isomorphism onto $\mathbb{H}$,

$$
\Pi_{2,0}^{\sharp}\left(\mathbb{V}^{0}\right)=\mathbb{H} \text {. }
$$

For every 1-form $\beta=\beta_{1,0}+\beta_{0,1}$, where $\beta_{1,0} \in \Gamma\left(\mathbb{V}^{0}\right)$ and $\beta_{0,1} \in \Gamma\left(\mathbb{H}^{0}\right)$, we have

$$
\Pi^{\sharp} \beta=\Pi_{2,0}^{\sharp} \beta_{1,0}+\Pi_{0,2}^{\sharp} \beta_{0,1} \text {. }
$$

Therefore, the characteristic distribution of $\Pi$ is the direct sum of the normal bundle $\mathbb{H}$, and the characteristic distribution of $\Pi_{0,2}$,

$$
\Pi^{\sharp}\left(T^{*} M\right)=\mathbb{H} \oplus \Pi_{0,2}^{\sharp}\left(\mathbb{H}^{0}\right) .
$$

This shows that the sets of singular points of the Poisson structures $\Pi$ and $\Pi_{0,2}$ coincide. Moreover, the symplectic leaves of $\Pi$ intersect the leaves of $\mathcal{F}$ transversally and symplectically. Notice also that $\Pi_{2,0}$ is a Poisson tensor if and only if the distribution $\mathbb{H}$ is integrable.

Now, given an $\mathcal{F}$-coupling Poisson structure $\Pi=\Pi_{2,0}+\Pi_{0,2}$ on $(M, \mathcal{F})$, we will assume that the action of a compact connected Lie group $G$ on $M$ is defined, such that it is compatible with the leaf-wise tangent Poisson tensor $\Pi_{0,2}$ in the sense that

$$
a_{M}=\Pi_{0,2}^{\sharp} \mu_{a}, \quad \forall a \in \mathfrak{g},
$$


for a certain $\mu=\mu_{1,0}+\mu_{0,1} \in \operatorname{Hom}\left(\mathfrak{g}, \Omega^{1}(M)\right)$. Let

$$
\Theta:=\delta^{G}\left(\mu_{0,1}\right),
$$

and consider the 2-forms on $M$

$$
B:=-\mathrm{d} \Theta, \quad B_{0,2}=-\mathrm{d}_{0,1} \Theta_{0,1} .
$$

Theorem 5.1. Let $S \subset M$ be a symplectic leaf of $\Pi$, such that

$$
T_{S} M=T S \oplus T_{S} \mathcal{F}
$$

Then, in a G-invariant open neighborhood, $N$, of $S$ in $M$, the Poisson tensor $\Pi$ is isomorphic to a $\mathcal{F}$-coupling Poisson tensor $\bar{\Pi}=\bar{\Pi}_{2,0}+\bar{\Pi}_{0,2}$, whose regular and singular components

$$
\bar{\Pi}_{2,0} \text { and } \bar{\Pi}_{0,2} \text { are } G \text {-invariant. }
$$

Around the leaf $S$, the Poisson structures $\bar{\Pi}$ and $\bar{\Pi}_{0,2}$ are related with $\Pi$ and $\Pi_{0,2}$ by the gauge transformations

$$
\begin{aligned}
& \bar{\Pi}^{\sharp}=\Pi^{\sharp} \circ\left(\mathrm{Id}-B^{\sharp} \circ \Pi^{\sharp}\right)^{-1}, \\
& \bar{\Pi}_{0,2}^{\sharp}=\Pi_{0,2}^{\sharp} \circ\left(\mathrm{Id}-B_{0,2}^{\sharp} \circ \Pi_{0,2}^{\sharp}\right)^{-1} .
\end{aligned}
$$

Proof. It follows from (5.3) and (5.4) that

$$
a_{M}=\Pi_{0,2}^{\sharp}\left(\mu_{a}\right)_{0,1}=\Pi^{\sharp}\left(\mu_{a}\right)_{0,1}
$$

and hence condition (3.5) holds for $\rho=\mu_{0,1}$. Therefore, the $G$-action is also compatible with $\Pi$. The transversality condition (5.5) says that $\Pi_{0,2}$ vanishes on $S$, and hence $\left.a_{M}\right|_{S}=0$. Then, by Theorem 4.1, in a $G$-invariant neighborhood $N$ of $S$, the gauge transformation (5.7) determines the $G$-invariant Poisson tensor $\bar{\Pi}$, which is isomorphic to $\Pi$ by a local diffeomorphism $\phi$ which restricts to the identity on $S$. Since the characteristic distributions of $\Pi$ and $\bar{\Pi}$ coincide on $N$, we conclude that $S$ is a symplectic leaf of $\bar{\Pi}$. Again by (5.5), one can choose the neighborhood $N$ (shrinking it, if needed) in such a way that $\overline{\mathbb{H}}:=\bar{\Pi}^{\sharp}\left(\mathbb{V}^{0}\right)$ is a normal bundle of $\mathcal{F}$, and hence $\bar{\Pi}=\bar{\Pi}_{2,0}+\bar{\Pi}_{0,2}$ is a $\mathcal{F}$-coupling Poisson tensor on $N$ (see, for example [23]). It follows from (5.4) that $a_{M} \in \Gamma(\mathbb{V})$, and hence we have the inclusions

$$
\llbracket \bar{\Pi}_{2,0}, a_{M} \rrbracket \in \chi^{2,0}(M) \oplus \chi^{1,1}(M), \quad \llbracket \bar{\Pi}_{0,2}, a_{M} \rrbracket \in \chi^{0,2}(M),
$$

where the bi-grading is taken with respect to the decomposition $T M=\overline{\mathbb{H}} \oplus \mathbb{V}$. These properties, and the $G$-invariance of $\bar{\Pi}$, imply that $\llbracket \bar{\Pi}_{2,0}, a_{M} \rrbracket=\llbracket \bar{\Pi}_{0,2}, a_{M} \rrbracket=0$, for every $a \in \mathfrak{g}$. This proves (5.6). Now, let us check (5.8). Consider the projection $\bar{p}_{V}: T M \rightarrow \mathbb{V}$, along $\overline{\mathbb{H}}$. Equality (5.7) reads

$$
\bar{\Pi}_{2,0}^{\sharp}+\bar{\Pi}_{0,2}^{\sharp}-\bar{\Pi}_{2,0}^{\sharp} \circ B^{\sharp} \circ \Pi^{\sharp}-\bar{\Pi}_{0,2}^{\sharp} \circ B^{\sharp} \circ \Pi^{\sharp}=\Pi_{2,0}^{\sharp}+\Pi_{0,2}^{\sharp},
$$

but taking into account the properties $\bar{\Pi}_{2,0}^{\sharp}\left(T^{*} M\right)=\overline{\mathbb{H}}$, and $\bar{\Pi}_{0,2}^{\sharp}\left(T^{*} M\right) \subset \mathbb{V}$, this equality is equivalent to the following relations, involving $\bar{p}_{V}$,

$$
\bar{\Pi}_{2,0}^{\sharp}-\bar{\Pi}_{2,0}^{\sharp} \circ B^{\sharp} \circ \Pi^{\sharp}=\left(\mathrm{id}-\bar{p}_{V}\right) \circ \Pi_{2,0}^{\sharp}, \quad \bar{\Pi}_{0,2}^{\sharp}-\bar{\Pi}_{0,2}^{\sharp} \circ B^{\sharp} \circ \Pi^{\sharp}=\bar{p}_{V} \circ \Pi_{2,0}^{\sharp}+\Pi_{02}^{\sharp} .
$$

As $\Pi_{2,0}^{\sharp}\left(\mathbb{H}^{0}\right)=0$, and $\bar{\Pi}_{0,2}^{\sharp}\left(\mathbb{V}^{0}\right)=\Pi_{0,2}^{\sharp}\left(\mathbb{V}^{0}\right)=0$, we conclude that the last equality splits into the following:

$$
\bar{\Pi}_{0,2}^{\sharp}-\bar{\Pi}_{0,2}^{\sharp} \circ B^{\sharp} \circ \Pi_{0,2}^{\sharp}=\Pi_{0,2}^{\sharp}, \quad \bar{\Pi}_{0,2}^{\sharp} \circ B^{\sharp} \circ \Pi_{2,0}^{\sharp}=-\bar{p}_{V} \circ \Pi_{2,0}^{\sharp} .
$$


By decomposing $B=B_{2,0}+B_{1,1}+B_{0,2}$, and using the properties $B_{2,0}^{\sharp}(\mathbb{V})=0$, and $B_{1,1}^{\sharp}(\mathbb{V}) \subset \mathbb{V}^{0}$, we get $\bar{\Pi}_{0,2}^{\sharp} \circ\left(B_{2,0}+B_{1,1}\right)^{\sharp} \circ \Pi_{0,2}^{\sharp}=0$. Hence $\bar{\Pi}_{0,2}^{\sharp}-\bar{\Pi}_{0,2}^{\sharp} \circ B_{0,2}^{\sharp} \circ \Pi_{0,2}^{\sharp}=\Pi_{02}^{\sharp}$. To finish, it suffices to notice that Id $+B_{0,2}^{\sharp} \circ \Pi_{0,2}^{\sharp}$ is invertible around the leaf $S$, because of the property $\Pi_{0,2}=0$ at $S$.

Corollary 5.2. The regular component of $\bar{\Pi}$ is given by

$$
\bar{\Pi}_{2,0}^{\sharp}=\left(\mathrm{id}-\bar{p}_{V}\right) \circ \Pi_{2,0}^{\sharp} \circ\left(\mathrm{Id}-B^{\sharp} \circ \Pi^{\sharp}\right)^{-1} .
$$

Remark 5.3. If the distribution $\mathbb{H}$ is integrable, then $\bar{\Pi}$ splits into two $G$-invariant Poisson structures: $\bar{\Pi}_{2,0}$, and $\bar{\Pi}_{0,2}$. Locally, around the fixed points of canonical group actions, such splitting always exists due to the equivariant versions of Weinstein splitting theorem [12, 17].

Now, consider the case of a $G$-action which is locally Hamiltonian on $\left(M, \Pi_{0,2}\right)$, that is, the compatibility condition (5.4) holds for a certain $\mu \in \operatorname{Hom}\left(\mathfrak{g}, \Omega_{\mathrm{cl}}^{1}(M)\right)$. That means $\mathrm{d} \mu_{a}=0$, for every $a \in \mathfrak{g}$, and the infinitesimal generator $a_{M}$ is locally Hamiltonian vector field on $\left(M, \Pi_{0,2}\right)$. Then, $0=\left(\mathrm{d} \mu_{a}\right)_{0,2}=\mathrm{d}_{0,1}\left(\mu_{a}\right)_{0,1}$, and hence $B_{0,2}=0$. Notice that the operator $\delta^{G}$ is compatible with the filtration given by $\Omega^{p, \bullet}$, so

$$
\delta^{G}\left(\operatorname{Hom}\left(\mathfrak{g}, \Omega^{p, q}(M)\right)\right) \subset \bigoplus_{k \geq 0} \Omega^{p+k, q-k}(M) .
$$

Moreover, since $\delta^{G}$ commutes with the exterior derivative, the 1 -form $\delta^{G}\left(\mu_{a}\right)$ is closed, and the gauge 2 -form in (5.7) can be represented as $B:=-\mathrm{d} Q$, where

$$
Q:=-\delta^{G}\left(\mu_{1,0}\right) \in \Omega^{1,0}(M)=\Gamma\left(\mathbb{V}^{0}\right) .
$$

Here we are using the property $\delta^{G}\left(\operatorname{Hom}\left(\mathfrak{g}, \Omega^{p, 0}(M)\right)\right) \subset \Omega^{p, 0}(M)$. It follows that $B=B_{2,0}+B_{1,1}$, with

$$
B_{2,0}=\mathrm{d}_{1,0} \circ \delta^{G}\left(\mu_{1,0}\right), \quad \text { and } \quad B_{1,1}=\mathrm{d}_{0,1} \circ \delta^{G}\left(\mu_{1,0}\right) .
$$

Thus, in this case Theorem 5.1 guarantees that, around the symplectic leaf $S, \Pi$ is Poissondiffeomorphic to the $G$-invariant $\mathcal{F}$-coupling Poisson tensor $\bar{\Pi}$, with $\bar{\Pi}_{0,2}=\Pi_{0,2}$.

In particular, if the action of the Lie group $G$ on $\left(M, \Pi_{0,2}\right)$ is Hamiltonian with momentum map $J \in \operatorname{Hom}\left(\mathfrak{g}, C^{\infty}(M)\right)$, so

$$
a_{M}=\Pi_{0,2}^{\sharp} \mathrm{d} J_{a}, \quad \forall a \in \mathfrak{g},
$$

then

$$
Q=-\delta^{G}\left(\mathrm{~d}_{1,0} J\right)
$$

For example, in the case $G=\mathbb{S}^{1}=\mathbb{R} \backslash 2 \pi \mathbb{Z}$, we have:

$$
Q=\frac{1}{2 \pi} \int_{0}^{2 \pi}(t-\pi)\left(\mathrm{Fl}_{\Pi_{0,2}^{\sharp} \mathrm{d} J}^{t}\right)^{*} \mathrm{~d}_{1,0} J \mathrm{~d} t-\pi\left\langle\mathrm{d}_{1,0} J\right\rangle .
$$

The adiabatic situation described in the following example, typically occurs in the theory of perturbations of Hamiltonian systems [1, 28].

Example 5.4. Let $M$ be a connected symplectic manifold (viewed as a parameter space), and let $\mathcal{P}$ be a Poisson manifold endowed with a smooth family of locally Hamiltonian actions $\Phi^{m}$ : $\mathcal{P} \times G \rightarrow \mathcal{P}$ (where $m \in M$ ), of a compact connected Lie group $G$. Let $x_{0} \in \mathcal{P}^{G}$ be a fixed point at which the Poisson structure on $\mathcal{P}$ has zero rank. Then, around the slice $M \times\left\{x_{0}\right\}$ (considered as a singular symplectic leaf), the product Poisson structure on $M \times \mathcal{P}$ is Poisson equivalent to the $G$-invariant Poisson tensor which gives rise to the averaged Hamiltonian dynamics. 


\section{Gauge transformations of geometric data}

In this section we describe a class of exact gauge transformations of coupling Dirac structures on a foliated manifold which preserve the coupling property.

\subsection{Connections on foliated manifolds}

Suppose we have a regular foliated manifold $(M, \mathcal{F})$. Let be $\mathbb{V}=T \mathcal{F}$ the tangent bundle, also called the vertical distribution. Recall that a vector valued 1-form $\gamma \in \Omega^{1}(M ; \mathbb{V})$ is a connection on $(M, \mathcal{F})$ if the vector bundle morphism $\gamma: T M \rightarrow \mathbb{V}$ satisfies the projection property $\gamma \circ \gamma=\gamma$, and $\operatorname{Im} \gamma=\mathbb{V}$. Then, $\mathbb{H}:=\operatorname{ker} \gamma$ is a normal bundle of $\mathcal{F}$, called the horizontal sub-bundle (with respect to the leaf space $M \backslash \mathcal{F}$ ). Reciprocally, given a normal bundle $\mathbb{H}$ of $\mathcal{F}$, one can define the associated connection as the projection $\gamma=p_{V}: T M \rightarrow \mathbb{V}$, according to the decomposition $T M=\mathbb{H} \oplus \mathbb{V}$.

The curvature of a connection $\gamma$ is the vector valued 2-form $R^{\gamma} \in \Omega^{2}(M ; \mathbb{V})$ on $M$ given by $R^{\gamma}=\frac{1}{2}[\gamma, \gamma]_{\mathrm{FN}}$. Here [, $]_{\mathrm{FN}}: \Omega^{k}(M ; T M) \times \Omega^{l}(M ; T M) \rightarrow \Omega^{k+l}(M ; T M)$ denotes the FrölicherNijenhuis bracket [15] of vector-valued forms on $M$. For example, for any $K, L \in \Omega^{1}(M ; T M)$, we have

$$
\begin{aligned}
{[K, L]_{\mathrm{FN}}(X, Y)=} & {[K X, L Y]-[K Y, L X]-L([K X, Y]-[K Y, X]) } \\
& -K([L X, Y]-[L Y, X])+(L K+K L)[X, Y],
\end{aligned}
$$

where $X, Y \in \mathfrak{X}(M)$.

Recall also that a vector field $X$ on $M$ is said to be projectable (on the leaf space $M \backslash \mathcal{F}$ ) if $[X, \Gamma(\mathbb{V})] \subset \Gamma(\mathbb{V})$. The space of all (local) projectable vector fields is denoted by $\chi_{\mathrm{pr}}(M, \mathcal{F})$. For a given connection $\gamma$, by $\Gamma_{\mathrm{pr}}(\mathbb{H})$ we denote the set of all (local) projectable sections of the horizontal subbundle $\mathbb{H}$. Then, the spaces $\chi(M)$ and $\Gamma(\mathbb{H})$ are locally generated by the elements of $\chi_{\mathrm{pr}}(M, \mathcal{F})$ and $\Gamma_{\mathrm{pr}}(\mathbb{H})$, respectively. In particular, the curvature of a connection $\gamma$ is uniquely determined by the relations

$$
R^{\gamma}(X, Y)=\gamma([X, Y]), \quad \forall X, Y \in \Gamma_{\mathrm{pr}}(\mathbb{H})
$$

and $\mathbf{i}_{V} R^{\gamma}=0$ for all $V \in \Gamma(\mathbb{V})$.

Fix a connection $\gamma$; then, any other connection $\tilde{\gamma}$ is of the form $\tilde{\gamma}=\gamma-\Xi$, where $\Xi \in \Omega^{1}(M ; \mathbb{V})$ is a vector valued 1 -form satisfying the condition $\mathbb{V} \subseteq$ Ker $\Xi$. The horizontal subbundle of $\tilde{\gamma}$ can be represented as

$$
\tilde{\mathbb{H}}=\operatorname{ker} \tilde{\gamma}=(\operatorname{Id}+\Xi)(\mathbb{H})
$$

Moreover, the transition rule for the curvature form reads

$$
R^{\tilde{\gamma}}=R^{\gamma}-\left([\gamma, \Xi]_{\mathrm{FN}}-\frac{1}{2}[\Xi, \Xi]_{\mathrm{FN}}\right) .
$$

Suppose now that the foliated manifold $(M, \mathcal{F})$ is endowed with a leaf-wise tangent Poisson bi-vector field $P \in \Gamma\left(\wedge^{2} \mathbb{V}\right)$. Then, each leaf of $\mathcal{F}$ inherits a Poisson structure from $P$ and we have a Poisson foliation denoted by $(M, \mathcal{F}, P)$. A connection $\gamma$ is said to be Poisson on $(M, \mathcal{F}, P)$ if every projectable section $X \in \Gamma_{\mathrm{pr}}(\mathbb{H})$ of the horizontal bundle $\mathbb{H}$ is a Poisson vector field on $(M, P)$. In this case, for every $X \in \Gamma_{\mathrm{pr}}(\mathbb{H}), R^{\gamma}(X, Y)$ is a vertical Poisson vector field. 


\subsection{Coupling Dirac structures}

By a set of geometric data on a foliated manifold $(M, \mathcal{F})$, we mean a triple $(\gamma, \sigma, P)$ consisting of a connection $\gamma \in \Omega^{1}(M ; \mathbb{V})$, a horizontal 2-form $\sigma \in \Gamma\left(\wedge^{2} \mathbb{V}^{0}\right)$ on $M$, and a leaf-wise tangent Poisson tensor $P \in \Gamma\left(\wedge^{2} \mathbb{V}\right)$. The geometric data $(\gamma, \sigma, P)$ are said to be integrable if they satisfy the structure equations

$$
\begin{aligned}
& \mathcal{L}_{X} P=0, \\
& \mathrm{~d}_{1,0}^{\gamma} \sigma=0, \\
& R^{\gamma}(X, Y)=-P^{\sharp} \mathrm{d} \sigma(X, Y),
\end{aligned}
$$

for any $X, Y \in \Gamma_{\text {pr }}(\mathbb{H})$. Here $\mathbb{H}=\operatorname{ker} \gamma$ is the horizontal sub-bundle and $\mathrm{d}_{1,0}^{\gamma}$ is the operator of bi-degree $(1,0)$, in the decomposition $(5.1)$, associated to $\mathbb{H}$. In particular, one has

$$
\mathrm{d}_{1,0}^{\gamma} \beta\left(X_{0}, X_{1}, \ldots, X_{q}\right)=\mathrm{d} \beta\left(X_{0}, X_{1}, \ldots, X_{q}\right)
$$

for any $\beta \in \Gamma\left(\wedge^{q} \mathbb{V}^{0}\right)$, and $X_{0}, X_{1}, \ldots, X_{q} \in \Gamma_{\mathrm{pr}}(\mathbb{H})$. Here $\mathrm{d}$ is the exterior differential on $M$. Conditions (6.4) and (6.5) say that $\gamma$ is a Poisson connection on $(M, \mathcal{F}, P)$, whose curvature takes values in the vertical Hamiltonian vector fields.

As is known $[23,25]$, every $\mathcal{F}$-coupling Poisson structure $\Pi$ on $(M, \mathcal{F})$ is equivalent to a set of integrable geometric data $(\gamma, \sigma, P)$, such that the restriction of $\sigma$ to $\mathbb{H}$ is non-degenerate, that is,

$$
\left.\sigma^{\sharp}\right|_{\mathbb{H}}: \mathbb{H} \rightarrow \mathbb{V}^{0} \text { is invertible. }
$$

The bi-vector field $\Pi$ can be reconstructed from $(\gamma, \sigma, P)$ by means of the formula $\Pi=\Pi_{2,0}+\Pi_{0,2}$, where $\Pi_{0,2}=P$, and $\Pi_{2,0} \in \Gamma\left(\wedge^{2} \mathbb{H}\right)$ is uniquely determined by the relation $\left.\Pi_{2,0}^{\sharp}\right|_{\mathbb{V} 0}=-\left(\left.\sigma^{\sharp}\right|_{\mathbb{H}}\right)^{-1}$. Therefore, the structure equations (6.4), (6.5), (6.6), give a factorization of the Jacobi identity for $\Pi$.

A Dirac structure $D \subset T M \oplus T^{*} M$ is said to be $\mathcal{F}$-coupling [24] if the associated tangent distribution $\mathbb{H}=\mathbb{H}(D, \mathcal{F})$,

$$
\mathbb{H}_{m}:=\left\{Z \in T_{m} M: \exists \alpha \in \mathbb{V}^{0} \text { and }(Z, \alpha) \in D\right\}
$$

is a normal bundle of $\mathcal{F}$. By lifting the non-degeneracy condition (6.8), we get the following fact $[11,24,29]$ : There exists a one-to-one correspondence $(\gamma, \sigma, P) \mapsto D$, between integrable geometric data and $\mathcal{F}$-coupling Dirac structures on $(M, \mathcal{F})$, which is given by

$$
D=\left\{\left(X+P^{\sharp} \alpha, \alpha-\mathbf{i}_{X} \sigma\right): X \in \Gamma(\mathbb{H}), \alpha \in \Gamma\left(\mathbb{H}^{0}\right)\right\}
$$

or, equivalently,

$$
D=\operatorname{Graph}\left(\left.\sigma\right|_{\mathbb{H}}\right) \oplus \operatorname{Graph}\left(\left.P\right|_{\mathbb{H}^{0}}\right) .
$$

The leaf-wise pre-symplectic structure associated to an $\mathcal{F}$-coupling Dirac structure $D$, can be described in terms of the corresponding geometric data as follows: Recall that the characteristic distribution $p_{T}(D)$, of $D$, is integrable, and gives rise to the singular pre-symplectic foliation $(\mathcal{S}, \omega)$, where $\omega$ is a leaf-wise pre-symplectic form. Then, $\mathcal{F} \cap \mathcal{S}$ is a symplectic foliation of $P$, and we have

$$
T \mathcal{S}=\mathbb{H} \oplus P^{\sharp}\left(\mathbb{V}^{0}\right) .
$$

This implies the point-wise splitting

$$
\omega_{m}=\sigma_{m} \oplus \tau_{m}, \quad \forall m \in M,
$$


where $\tau$ is the leaf-wise symplectic form associated to $P$. It follows that $T \mathcal{S} \cap \mathbb{V}$ is the characteristic distribution of $P$, and in terms of the pre-symplectic form, the characteristic sub-bundle of the $\mathcal{F}$-coupling Dirac structure $D$, is represented as

$$
\mathbb{H}_{m}=\left(T_{m} \mathcal{S} \cap \mathbb{V}_{m}\right)^{\omega} \equiv\left\{X \in T_{m} \mathcal{S}: \omega_{m}\left(X, P^{\sharp} d f\right)=0, \forall f \in C_{\mathrm{loc}}^{\infty}(M)\right\} .
$$

It is useful to rewrite condition (3.1) (for a vector field $X$ on $M$ to be Hamiltonian, relative to the $\mathcal{F}$-coupling Dirac structure $D)$ in terms of the geometric data $(\gamma, \sigma, P)$. It easy to see that the vector field $X=X_{1,0}+X_{0,1}$ is Hamiltonian on $(M, D)$ if and only if the components $X_{1,0} \in \Gamma(\mathbb{H})$, and $X_{0,1} \in \Gamma(\mathbb{V})$, satisfy the relations:

$$
\begin{aligned}
& X_{0,1}=P^{\sharp} \mathrm{d} F, \\
& \mathbf{i}_{X_{1,0}} \sigma=-\mathrm{d}_{1,0}^{\gamma} F,
\end{aligned}
$$

for a certain $F \in C^{\infty}(M)$.

We remark that there is a natural class of coupling Dirac structures on vector bundles, which comes from transitive Lie algebroids and plays an important rôle in constructing linearized models around (pre) symplectic leaves of Poisson and Dirac manifolds [9, 24, 25].

\section{3 $Q$-gauge transformations}

Here, we will describe some symmetries of the structure equations (see also [26]). Let $(\gamma, \sigma, P)$ be some geometric data on $(M, \mathcal{F})$ and $Q \in \Gamma\left(\mathbb{V}^{0}\right)$ a horizontal 1-form. For every $\beta \in \Gamma\left(\wedge^{q} \mathbb{V}^{0}\right)$, denote by $\{Q \wedge \beta\}_{P}$ the element of $\Gamma\left(\wedge^{q+1} \mathbb{V}^{0}\right)$ given by

$$
\{Q \wedge \beta\}_{P}\left(X_{0}, X_{1}, \ldots, X_{q}\right):=\sum_{i=0}^{q}(-1)^{i}\left\{Q\left(X_{i}\right), \beta\left(X_{0}, X_{1}, \ldots, \hat{X}_{i}, \ldots, X_{q}\right)\right\}_{P},
$$

where $\left\{f_{1}, f_{2}\right\}_{P}=P\left(\mathrm{~d} f_{1}, \mathrm{~d} f_{2}\right)$ is the Poisson bracket associated to $P$. Define

$$
\begin{aligned}
& \tilde{\gamma}:=\gamma-\Xi^{Q}, \\
& \tilde{\sigma}:=\sigma-\left(\mathrm{d}_{1,0}^{\gamma} Q+\frac{1}{2}\{Q \wedge Q\}_{P}\right),
\end{aligned}
$$

where $\Xi^{Q} \in \Omega^{1}(M ; \mathbb{V})$ is the vector-valued 1-form uniquely determined by the condition $\Xi^{Q}(X)=$ $P^{\sharp} \mathrm{d} Q(X)$, for every $X \in \chi_{\mathrm{pr}}(M, \mathcal{F})$. Evidently, the vector-valued 1-form $\tilde{\gamma}$ determines a connection on $(M, \mathcal{F})$, and $\tilde{\sigma} \in \Gamma\left(\wedge^{2} \mathbb{V}^{0}\right)$. One can think of the mapping $(\gamma, \sigma, P) \mapsto(\tilde{\gamma}, \tilde{\sigma}, P)$ as a gauge transformation defined on the set of all geometric data on $(M, \mathcal{F})$, leaving fixed the Poisson tensor $P$. The following result shows that such gauge transformations preserve the coupling property.

Proposition 6.1. Let $D$ be a $\mathcal{F}$-coupling Dirac structure, associated to the integrable geometric data $(\gamma, \sigma, P)$ on $(M, \mathcal{F})$, and let $Q \in \Gamma\left(\mathbb{V}^{0}\right)$ be an arbitrary horizontal 1-form on $M$. Then, the triple $(\tilde{\gamma}, \tilde{\sigma}, P)$ defined by (6.14), (6.15), satisfies the structure equations (6.4) to (6.6). Moreover, the $\mathcal{F}$-coupling Dirac structure $\tilde{D}$, associated to the integrable geometric data $(\tilde{\gamma}, \tilde{\sigma}, P)$, is related to $D$ by the exact gauge transformation:

$$
\tilde{D}=\left\{\left(X, \alpha-\mathbf{i}_{X} \mathrm{~d} Q\right):(X, \alpha) \in D\right\} .
$$

Proof. Let $\tilde{\mathbb{H}}=\operatorname{ker} \tilde{\gamma}$ be the horizontal bundle of $\tilde{\gamma}$. From (6.2) and (6.14), we get that every projectable vector field $\tilde{X} \in \Gamma_{\mathrm{pr}}(\tilde{\mathbb{H}})$ can be represented as

$$
\tilde{X}=X+P^{\sharp} \mathrm{d} Q(X), \quad X \in \Gamma_{\mathrm{pr}}(\mathbb{H}),
$$


and hence $\tilde{X}$ is a Poisson vector field with respect to $P$. That the curvature identity (6.6) for $R^{\tilde{\gamma}}$ is satisfied, can be straightforwardly checked, by using the fact that $\gamma$ is a Poisson connection, the equality $\gamma(\tilde{X})=\Xi^{Q}(\tilde{X})=P^{\sharp} \mathrm{d} Q(X)$, and relations (6.1), (6.3). The corresponding coupling form $\tilde{\sigma}$ is just given by (6.15). The structure equations for $(\gamma, \sigma, P)$ imply the following identities:

$$
\left(\mathrm{d}_{1,0}^{\gamma}\right)^{2} Q=\{Q \wedge \sigma\}_{P}, \quad \mathrm{~d}_{1,0}^{\gamma}\{Q \wedge Q\}_{P}=-2\left\{Q \wedge \mathrm{d}_{1,0}^{\tilde{\gamma}} Q\right\}_{P} .
$$

Moreover, by (6.14), we have

$$
\mathrm{d}_{1,0}^{\tilde{\gamma}} \beta=\mathrm{d}_{1,0}^{\gamma} \beta+\{Q \wedge \beta\}_{P}, \quad \beta \in \Gamma\left(\wedge^{q} \mathbb{V}^{0}\right) .
$$

Using these relations, it can be readily checked that $\mathrm{d}_{1,0}^{\tilde{\gamma}} \tilde{\sigma}=0$. This proves the integrability of $(\tilde{\gamma}, \tilde{\sigma}, P)$. Now, consider the Dirac structure $\tilde{D}$, induced by $(\tilde{\gamma}, \tilde{\sigma}, P)$. Relations (6.10) and (6.17), show that $p_{T}(\tilde{D})=p_{T}(D)$. Let $(\mathcal{S}, \tilde{\omega})$ and $(\mathcal{S}, \omega)$ be the pre-symplectic foliations associated to $\tilde{D}$ and $D$, respectively. Then, $T \mathcal{S}$ is generated by local projectable vector fields of the form (6.17), and $P^{\sharp} \mathrm{d} f$, where $X \in \Gamma_{\mathrm{pr}}(\mathbb{H})$, and $f \in C_{\mathrm{loc}}^{\infty}(M)$. Evaluating the pre-symplectic forms $\tilde{\omega}$ and $\omega$ on this family of vector fields, and using the point-wise splitting (6.11) for $\tilde{\omega}$, we can verify, by a straightforward computation, that

$$
\tilde{\omega}_{S}+\iota_{S}^{*} \mathrm{~d} Q=\omega_{S}
$$

at every pre-symplectic leaf $S$ of $\mathcal{S}$. This means that $\tilde{D}$ is given by (6.16).

Therefore, gauge transformations of integrable geometric data lead to exact gauge transformations of Dirac structures. The reciprocal is also true.

Proposition 6.2. For every $Q \in \Gamma\left(\mathbb{V}^{0}\right)$ and an $\mathcal{F}$-coupling Dirac structure $D$, the exact gauge transformation (6.16) takes $D$ to the $\mathcal{F}$-coupling Dirac structure $\tilde{D}$, whose geometric data are given by (6.14), (6.15).

Proof. Let us show first that $\tilde{D}$ is $\mathcal{F}$-coupling. The Dirac structures $\tilde{D}$ and $D$ determine the same leaf partition $\mathcal{S}$ of $M$, and the corresponding pre-symplectic structures $\tilde{\omega}$ and $\omega$, are related by (6.18). Because of (6.10), any vector field $X \in \Gamma(\mathbb{H})$ and Hamiltonian vector field $P^{\sharp} \mathrm{d} f$, are tangent to the foliation $\mathcal{S}$, and $\omega$-orthogonal. Then, any arbitrary projectable vector field $\tilde{X}$, of the form (6.17), and $P^{\sharp} \mathrm{d} f$ are $\tilde{\omega}$-orthogonal,

$$
\tilde{\omega}\left(\tilde{X}, P^{\sharp} \mathrm{d} f\right)=\tau\left(P^{\sharp} \mathrm{d} Q(X), P^{\sharp} \mathrm{d} f\right)-\mathrm{d} Q\left(\tilde{X}, P^{\sharp} \mathrm{d} f\right)=\{Q(X), f\}_{P}+\mathcal{L}_{P^{\sharp} \mathrm{d} f} Q(X)=0 .
$$

According to (6.17), the tangent distribution (6.9), associated to $\tilde{D}$, is given by

$$
\tilde{\mathbb{H}}=\operatorname{Span}\left\{\tilde{X}=X+P^{\sharp} \mathrm{d} Q(X): X \in \Gamma_{\operatorname{pr}}(\mathbb{H})\right\},
$$

and hence it is a normal bundle of $\mathcal{F}$. Therefore, $\tilde{D}$ is a $\mathcal{F}$-coupling Dirac structure. Let $(\tilde{\gamma}, \tilde{\sigma}, \tilde{P})$ be the corresponding integrable geometric data. The connection $\tilde{\gamma}$, induced by $\tilde{\mathbb{H}}$, is given by (6.14). Moreover, by (6.18) and the condition that $Q$ is horizontal, we conclude that the restriction of $\tilde{\omega}$ to $T_{m} \mathcal{S} \cap \mathbb{V}_{m}$ coincides with $\tau_{m}$. Thus, $\tilde{P}=P$. Finally, using (6.18) and (6.19), we compute the coupling 2-form $\tilde{\sigma}$

$$
\begin{aligned}
\tilde{\sigma}\left(\tilde{X}_{1}, \tilde{X}_{2}\right) & =\tilde{\omega}\left(\tilde{X}_{1}, \tilde{X}_{2}\right)=\omega\left(\tilde{X}_{1}, \tilde{X}_{2}\right)-\mathrm{d} Q\left(\tilde{X}_{1}, \tilde{X}_{2}\right) \\
& =\sigma\left(X_{1}, X_{2}\right)+\left\{Q\left(X_{1}\right), Q\left(X_{2}\right)\right\}_{P}-\mathrm{d} Q\left(X_{1}, X_{2}\right)-2\left\{Q\left(X_{1}\right), Q\left(X_{2}\right)\right\}_{P} \\
& =\sigma\left(X_{1}, X_{2}\right)-\mathrm{d} Q\left(X_{1}, X_{2}\right)-\left\{Q\left(X_{1}\right), Q\left(X_{2}\right)\right\}_{P} .
\end{aligned}
$$

Therefore, $\tilde{\sigma}$ is just given by (6.15).

Remark 6.3. Gauge transformations of the form (6.14), (6.15), appear naturally in the classification theory of Poisson structures around a symplectic leaf [26], and in the gauge theory on principal bundles [10]. 


\section{$7 \quad$ Averaging of coupling Dirac structures}

In this section we present a generalization of some results obtained in [27] in the case of Hamiltonian actions on Poisson fiber bundles. This time, without the requirement of the existence of a global momentum map, we describe the averaging procedure for coupling Dirac (not just Poisson) structures on a foliated manifold with respect to a class of locally Hamiltonian group actions.

Suppose we have an action $\Phi: G \times M \rightarrow M$, of a compact connected Lie group $G$ on a foliated manifold $(M, \mathcal{F})$, which preserves the foliation, $\left(\Phi_{g}\right)_{* m} \mathbb{V}_{m}=\mathbb{V}_{\Phi_{g}(m)}$, for all $g \in G$. It is clear the pull-back $\Phi_{g}^{*}$ preserves the subspaces $\Omega^{p}(M ; \mathbb{V}) \subset \Omega^{p}(M ; T M)$, and hence the averaging operator $\langle\cdot\rangle^{G}: \Omega^{p}(M ; \mathbb{V}) \rightarrow \Omega^{p}(M ; \mathbb{V})$ is well-defined on vector valued forms through

$$
\langle K\rangle^{G}(X)=\int_{G} \Phi_{g}^{*}\left(K\left(\left(\Phi_{g}\right)_{*} X\right) \mathrm{d} g,\right.
$$

for every $K \in \Omega^{p}(M ; \mathbb{V}), X \in \chi(M)$. Notice that $K$ is $G$-invariant if and only if $K=\langle K\rangle^{G}$.

In particular, by averaging a connection $\gamma$ we obtain a $G$-invariant connection $\langle\gamma\rangle^{G}$. Indeed, taking into account that the $G$-action preserves the subspace of vertical vector fields, it easy to see that $\langle\gamma\rangle^{G}(V)=V$, for all $V \in \Gamma(\mathbb{V})$. The difference vector 1 -form $\Xi:=\gamma-\langle\gamma\rangle^{G} \in \Omega^{1}(M ; \mathbb{V})$ has zero average, $\langle\Xi\rangle^{G}=0$, and admits the representation $\Xi=\delta^{G} \circ l^{G}(\gamma)$. Here the $\mathbb{R}$-linear mapping $l^{G}: \Omega^{1}(M ; \mathbb{V}) \rightarrow \operatorname{Hom}\left(\mathfrak{g} ; \Omega^{1}(M ; \mathbb{V})\right)$ is defined by $l^{G}(\gamma)_{a}=\left[a_{M}, \gamma\right]_{\mathrm{FN}}$. The horizontal bundle $\overline{\mathbb{H}}=(\operatorname{Id}+\Xi)(\mathbb{H})$ of $\langle\gamma\rangle^{G}$, and the curvature form $R^{\langle\gamma\rangle^{G}}$, are also $G$-invariant.

\subsection{G-invariant integrable geometric data}

As we have seen in Sections 2 and 6, the averaging procedure for Dirac structures is well-defined with respect to the class of compatible compact group actions, and is related to the existence exact gauge transformations. Here we show that the $G$-average $\bar{D}=D^{\langle\omega\rangle^{G}}$ of a $\mathcal{F}$-coupling Dirac structure $D$, with respect to a locally Hamiltonian $G$-action, inherits the coupling property and give computational formulae for the corresponding invariant geometric data.

First, we observe that given a foliation-preserving action $\Phi: G \times M \rightarrow M$, of a Lie group $G$ on $(M, \mathcal{F})$, we have an induced $G$-action on the set of all geometric data on $(M, \mathcal{F})$, defined by the transformations

$$
(\gamma, \sigma, P) \mapsto\left(\Phi_{g}^{*} \gamma, \Phi_{g}^{*} \sigma, \Phi_{g}^{*} P\right) .
$$

It is easy to see that these transformations are symmetries of the structures equations (6.4) to (6.6). In other words, the induced action preserves the subset of integrable geometric data. Recall that a Dirac structure $D$ is $G$-invariant if, for any $(\alpha, X) \in \Gamma(D)$ and $g \in G$, we have $\left(\Phi_{g}^{*} \alpha, \Phi_{g}^{*} X\right) \in \Gamma(D)$. Then, it is possible to show that an $\mathcal{F}$-coupling Dirac structure is invariant, with respect to the $G$-action on $(M, \mathcal{F})$, if and only if the associated integrable geometric data $(\gamma, \sigma, P)$ are $G$-invariant, that is, invariant with respect to the induced $G$-action $[24,26]$.

Theorem 7.1. Let $D$ be a $\mathcal{F}$-coupling Dirac structure on $(M, \mathcal{F})$, associated to the integrable geometric data $(\gamma, \sigma, P)$. Let $\Phi: G \times M \rightarrow M$ be a locally Hamiltonian action of a compact connected Lie group $G$ on $(M, \mathcal{F}, P)$,

$$
a_{M}=P^{\sharp} \mu_{a}, \quad \mathrm{~d} \mu_{a}=0 .
$$

Then, the G-average $\bar{D}=D^{\langle\omega\rangle^{G}}$ of $D$, is an $\mathcal{F}$-coupling Dirac structure on $(M, \mathcal{F})$, associated to the $G$-invariant geometric data $(\bar{\gamma}, \bar{\sigma}, P)$,

$$
\bar{D}=\operatorname{Graph}\left(\left.\bar{\sigma}\right|_{\overline{\mathbb{H}}}\right) \oplus \operatorname{Graph}\left(\left.P\right|_{\overline{\mathbb{H}}^{0}}\right),
$$


which are given by

$$
\begin{aligned}
& \bar{\gamma}:=\langle\gamma\rangle^{G} \equiv \gamma-\Xi^{Q}, \\
& \bar{\sigma}:=\langle\sigma\rangle^{G}+\frac{1}{2}\left\langle\{Q \wedge Q\}_{P}\right\rangle^{G}-\mathrm{d}_{1,0}^{\bar{\gamma}}\langle Q\rangle^{G} .
\end{aligned}
$$

Here

$$
Q=-\delta^{G}\left(\mu_{1,0}\right) \in \Gamma\left(\mathbb{V}^{0}\right) .
$$

Proof. It follows from (7.1) that the locally Hamiltonian $G$-action is compatible with $D$, and hence, by Proposition 3.1, the average $D^{\langle\omega\rangle^{G}}$ is well-defined and related to $D$ by the exact gauge transformation (6.16), where the horizontal 1-form $Q$ is given by (7.5). Then, by Proposition 6.2, $D^{\langle\omega\rangle^{G}}$ is an $\mathcal{F}$-coupling Dirac structure associated to the geometric data $(\bar{\gamma}, \bar{\sigma}, P)$, where $\bar{\gamma}$ is given by (7.3), and

$$
\bar{\sigma}:=\sigma-\left(\mathrm{d}_{1,0}^{\gamma} Q+\frac{1}{2}\{Q \wedge Q\}_{P}\right) .
$$

Since the averaged Dirac structure is invariant with respect to the $G$-action, the data $(\bar{\gamma}, \bar{\sigma})$ are also $G$-invariant. Averaging (7.3), and the identity

$$
\left(\mathrm{d}_{1,0}^{\bar{\gamma}} Q\right)=\mathrm{d}_{1,0}^{\gamma} Q+\{Q \wedge Q\}_{P}
$$

we get the relations

$$
\bar{\sigma}=\langle\bar{\sigma}\rangle^{G}=\langle\sigma\rangle^{G}-\left\langle\mathrm{d}_{1,0}^{\gamma} Q\right\rangle^{G}-\frac{1}{2}\left\langle\{Q \wedge Q\}_{P}\right\rangle^{G},
$$

and

$$
\left\langle\mathrm{d}_{1,0}^{\gamma} Q\right\rangle^{G}=\mathrm{d}_{1,0}^{\bar{\gamma}}\langle Q\rangle^{G}+\left\langle\{Q \wedge Q\}_{P}\right\rangle^{G}
$$

This proves (7.4).

As a consequence of this result, we have the following alternative version of Theorem 5.1.

Corollary 7.2. Under the hypotheses of Theorem 7.1, suppose that $D=$ Graph $\Pi$ is the graph of a Poisson tensor $\Pi$ on $M$, which has a symplectic leaf $S$ satisfying the transversality condition (5.5). Then, in a neighborhood of $S$, we have $\bar{D}=$ Graph $\bar{\Pi}$, where $\bar{\Pi}=\bar{\Pi}_{2,0}+P$ is a G-invariant coupling Poisson tensor, whose geometric data are given by (7.3) and (7.4). In particular, the G-invariant component $\bar{\Pi}_{2,0}$ is defined by (cf. (5.9)):

$$
\left.\bar{\Pi}_{2,0}^{\sharp}\right|_{\mathbb{V} 0}=-\left(\left.\bar{\sigma}^{\sharp}\right|_{\overline{\mathbb{H}}}\right)^{-1} \text {. }
$$

In terms of the geometric data, a Poisson diffeomorphism $\phi$, between the Poisson structures $\bar{\Pi}$ and $\Pi$, can be constructed in the following way [26]: Consider the family of integrable geometric data $\left(\gamma_{t}, \sigma_{t}, P\right)$, defined by $\gamma_{t}=\gamma-t \Xi^{Q}$, and

$$
\sigma_{t}=\sigma-\left(t \mathrm{~d}_{1,0}^{\gamma} Q+\frac{t^{2}}{2}\{Q \wedge Q\}_{P}\right)
$$

Because of the transversality condition, in a neighborhood of $S,\left.\sigma_{t}\right|_{H_{t}}$ is non-degenerate for all $t \in[0,1]$. As a consequence, there exists a unique time-dependent vector field $Z_{t} \in \Gamma\left(\mathbb{H}_{t}\right)$ satisfying the equation $\mathbf{i}_{Z_{t}} \sigma_{t}=Q$. Then, $\phi$ is defined by evaluating the flow of $Z_{t}$ at time $t=1$. 


\subsection{Invariant sections of $\bar{D}$}

This brief subsection is devoted to some remarks about invariant sections of the averaged Dirac structure $\bar{D}$. First, notice that the $G$-invariant sections of the horizontal bundle $\overline{\mathbb{H}}$ of the averaged Poisson connection $\bar{\gamma}(7.3)$, can be described in the following way. Let $X \in \Gamma_{\mathrm{pr}}(\mathbb{H})$ be a projectable section of $\mathbb{H}$, defined on an invariant domain of $M$. Then, the $G$-average $\langle X\rangle^{G}$ is a projectable section of $\overline{\mathbb{H}}$, of the form

$$
\langle X\rangle^{G}=X+P^{\sharp} \mathrm{d} Q(X) \in \Gamma_{\mathrm{pr}}(\overline{\mathbb{H}}),
$$

where $Q$ is given by (7.5). It follows that

$$
\left(X+P^{\sharp} \mathrm{d} Q(X),-\mathbf{i}_{X} \bar{\sigma}\right)
$$

is a $G$-invariant section of the Dirac structure $\bar{D}$. Moreover, the sub-bundle $\overline{\mathbb{H}}^{0} \subset T^{*} M$ is invariant under the action of $G$, and every $G$-invariant 1-form $\beta \in \Gamma\left(\overline{\mathbb{H}}^{0}\right)$ induces the $G$-invariant section

$$
\left(P^{\sharp} \beta, \beta\right)
$$

of $\bar{D}$. However, notice that in general these sections do not generate $\bar{D}$; in the following subsection we will consider a case where they do. On the other hand, it can be shown [19] that the Dirac structure $\bar{D}$ is locally spanned by $G$-invariant sections; this fact is based on the tube theorem and the averaging procedure for proper Lie group actions $[13,14]$.

\subsection{Hamiltonian actions}

Below we will assume that the hypotheses of Theorem 7.1 hold, and the foliation $\mathcal{F}$ is a fibration. Therefore, the leaf space $B=M \backslash \mathcal{F}$ is a smooth manifold and the natural projection $\pi: M \rightarrow B$ is a submersion, which we will assume has connected fibers. In this case, every projectable section $X \in \Gamma_{\mathrm{pr}}(\mathbb{H})$ is the $\gamma$-horizontal lift of a smooth vector field on $B$ and, hence, it is welldefined on a $G$-invariant open domain of $M$. This implies the following important property: The horizontal bundle $\overline{\mathbb{H}}$ of $\bar{\gamma}$ is spanned by $G$-invariant Poisson vector fields of the form (7.5). As a consequence, we also get that the averaged Dirac structure $\bar{D}$ is spanned by $G$-invariant sections of the form (7.7) and (7.8).

Now, suppose that the action of the Lie group $G$ on $(M, P)$ is Hamiltonian, with momentum $\operatorname{map} J \in \operatorname{Hom}\left(\mathfrak{g} ; C^{\infty}(M)\right)$,

$$
a_{M}=P^{\sharp} \mathrm{d} J_{a}, \quad \forall a \in \mathfrak{g} .
$$

In general, the $G$-action is not Hamiltonian with respect to the original coupling Dirac structure $D$. As it follows from (6.12), (6.13), this happens only in the particular case $\mathrm{d}_{1,0}^{\gamma} J=0$. Thus, it is natural to ask whether the $G$-action is Hamiltonian with respect to the averaged Dirac structure $\bar{D}$ (7.2). The key property in this regard is that, for every $X \in \Gamma_{\mathrm{pr}}(\mathbb{H})$, we have

$$
\left\langle\mathcal{L}_{X} J_{a}\right\rangle^{G}=\mathcal{L}_{\langle X\rangle^{G}} J_{a} \in \operatorname{Casim}(M ; P),
$$

where $\operatorname{Casim}(M ; P)$ denotes the space of Casimir functions of $P$. Indeed, noticing first that

$$
P^{\sharp} \mathrm{d}\left(\mathcal{L}_{\langle X\rangle^{G}} J_{a}\right)=\left[\langle X\rangle^{G}, P^{\sharp} \mathrm{d} J_{a}\right]=0,
$$

we can use (7.6) to get

$$
\mathcal{L}_{\langle X\rangle^{G}} J_{a}=\mathcal{L}_{X} J_{a}+\left\{Q(X), J_{a}\right\}_{P}=\mathcal{L}_{X} J_{a}-\mathcal{L}_{a_{M}} Q(X) .
$$


Averaging this equality, and taking into account that every Casimir function is $G$-invariant, we obtain

$$
\mathcal{L}_{\langle X\rangle^{G}} J_{a}=\left\langle\mathcal{L}_{\langle X\rangle^{G}} J_{a}\right\rangle^{G}=\left\langle\mathcal{L}_{X} J_{a}\right\rangle^{G}-\left\langle\mathcal{L}_{a_{M}} Q(X)\right\rangle^{G}=\left\langle\mathcal{L}_{X} J_{a}\right\rangle^{G} .
$$

This proves (7.9). Moreover, since the group $G$ acts along the fibers of the projection $\pi$, the averaging operator preserves the subspace of horizontal 1-forms. In particular, $\left\langle\mathrm{d}_{1,0}^{\gamma} J_{a}\right\rangle^{G} \in$ $\Gamma\left(\mathbb{V}^{0}\right)$. Using this fact and properties (6.7), (7.6) and (7.9), we get

$$
\begin{aligned}
\mathbf{i}_{\langle X\rangle^{G}} \mathrm{~d}_{1,0}^{\bar{\gamma}} J_{a} & =\mathbf{i}_{\langle X\rangle^{G}} \mathrm{~d}^{\bar{\gamma}} J_{a}=\mathbf{i}_{\langle X\rangle^{G}} \mathrm{~d} J_{a}=\mathcal{L}_{\langle X\rangle^{G}} J_{a}=\left\langle\mathcal{L}_{X} J_{a}\right\rangle^{G} \\
& =\left\langle\mathbf{i}_{\langle X\rangle^{G}} \mathrm{~d}_{1,0}^{\gamma} J_{a}\right\rangle^{G}=\mathbf{i}_{X}\left\langle\mathrm{~d}_{1,0}^{\gamma} J_{a}\right\rangle^{G},
\end{aligned}
$$

for every $X \in \Gamma_{\mathrm{pr}}(\mathbb{H})$.

In this way, we arrive at the following criterion.

Proposition 7.3. The G-action is Hamiltonian on the Dirac manifold $(M, \bar{D})$, with momentum map $J$,

$$
\left(a_{M}, \mathrm{~d} J_{a}\right) \in \Gamma(\bar{D}), \quad \forall a \in \mathfrak{g},
$$

if and only if

$$
\left\langle\mathrm{d}_{1,0}^{\gamma} J\right\rangle^{G}=0
$$

Proof. It follows from (6.12), (6.13), that the infinitesimal generator $a_{M}$ of the $G$-action is Hamiltonian, relative to the Dirac structure $\bar{D}$ and the function $J_{a}$, if and only if $\mathrm{d}_{1,0}^{\bar{\gamma}} J_{a}=0$. This equality, together with (7.10), implies (7.11).

Therefore, condition (7.11) (known as the "adiabatic hypothesis" in the theory of HannayBerry connections on Poisson fiber bundles [16]), appears in the context of the Hamiltonization of the $G$-action with respect to the Dirac structure $\bar{D}$. The freedom in the choice of the momentum map is given by the transformation $J \mapsto J-K$, for arbitary $K \in \operatorname{Hom}(\mathfrak{g} ; \operatorname{Casim}(M ; P))$. Fixing $J$, the point is to choose $K$ in such a way that $J-K$ satisfies (7.11). This question can be reformulated in cohomological terms. Let $\mathcal{C}_{B}^{k}:=\Omega^{k}(B) \otimes \operatorname{Casim}(M ; P)$ be the space of $k$ forms on the base $B$, with values in the algebra of Casimir functions of the Poisson structure $P$. Consider the operator $\partial: \mathcal{C}_{B}^{k} \rightarrow \mathcal{C}_{B}^{k+1}$ defined by $(\partial \beta)\left(u_{1}, \ldots, u_{k}\right)=\mathrm{d}\left(\pi^{*} \beta\right)\left(X_{1}, \ldots, X_{k}\right)$. Here, $X_{i} \in \Gamma_{\mathrm{pr}}(\mathbb{H})$ is the $\gamma$-horizontal lift of a vector field $u_{i}$ on $B$, and $\pi^{*}: \mathcal{C}_{B}^{k} \rightarrow \Gamma\left(\wedge^{k} \mathbb{V}^{0}\right)$ is the pull-back. Then, it follows from the curvature identity (6.6) that $\partial$ is a coboundary operator, $\partial^{2}=0[26]$. Property (7.9) implies

$$
\left\langle\mathrm{d}_{1,0}^{\gamma} J\right\rangle^{G}=\pi^{*} \zeta
$$

for a certain $\zeta \in \operatorname{Hom}\left(\mathfrak{g} ; \mathcal{C}_{B}^{1}\right)$. Moreover one can prove [16] that, for all $X, Y \in \Gamma_{\operatorname{pr}}(\mathbb{H})$,

$$
\mathbf{i}_{X} \mathbf{i}_{Y} \mathrm{~d}\left\langle\mathrm{~d}_{1,0}^{\gamma} J_{a}\right\rangle^{G}=0
$$

and hence $\zeta_{a} \in \mathcal{C}_{B}^{k}$ is a $\partial$-closed 1-form, for every $a \in \mathfrak{g}$. Finally, we conclude that the $G$-action admits a momentum map satisfying (7.11) if and only if the $\partial$-cohomology class of $\zeta$ is trivial.

Example 7.4. Consider the particular case in which the Poisson bundle $(\pi: M \rightarrow B, P)$, associated to the original coupling Dirac structure $D$, is a locally trivial Lie-Poisson bundle over $B$ with typical fiber $\mathfrak{h}^{*}$, the dual of a Lie algebra $\mathfrak{h}$. Therefore, the restriction of $P$ to each fiber of $\pi$ is isomorphic to the Lie-Poisson structure on the co-algebra $\mathfrak{h}^{*}$. Assume also 
that the Poisson connection $\gamma$ is homogeneous in the sense that the Lie derivative along every $X \in \Gamma_{\mathrm{pr}}(\mathbb{H})$, preserves the space of fiber-wise linear functions $C_{\operatorname{lin}}^{\infty}(M)$ on the total space of $\pi$. Finally, suppose that the momentum map $J$ of the Hamiltonian $G$-action on $(M, P)$, is fiberwise linear, $J \in C_{\operatorname{lin}}^{\infty}(M)$. It follows that, for every $a \in \mathfrak{h}$, the 1 -form $\zeta_{a}$ on $B$ takes values in the fiber-wise linear Casimir functions of $P$. Our remark is that if the center is trivial, $Z(\mathfrak{h})=\{0\}$, then $\zeta=0$, and hence the $G$-action is Hamiltonian relative to the Dirac structure $\bar{D}$, with the same momentum map $J$.

Notice also that, in the case in which the Casimir functions of $P$ can be described as pull-backs of functions on the base, $\operatorname{Casim}(M ; P)=\pi^{*} C^{\infty}(B)$, the cohomology class of $\zeta$ is trivial if $B$ is simply connected. This setting appears in the symplectic case, where the Poisson structure $P$ is non degenerate (see also [16]).

\section{Acknowledgements}

The first author (JAV) was partially supported by the Mexican Consejo Nacional de Ciencia y Tecnología (CONACyT) research project CB-2012 179115. Both authors acknowledge the detailed comments of the referees, which helped to improve the contents and presentation of this paper.

\section{References}

[1] Avendaño-Camacho M., Vallejo J.A., Vorobiev Yu., Higher order corrections to adiabatic invariants of generalized slow-fast Hamiltonian systems, J. Math. Phys. 54 (2013), 082704, 15 pages, arXiv:1305.3974.

[2] Brahic O., Fernandes R.L., Poisson fibrations and fibered symplectic groupoids, in Poisson geometry in mathematics and physics, Contemp. Math., Vol. 450, Amer. Math. Soc., Providence, RI, 2008, 41-59, math.DG/0702258.

[3] Brahic O., Fernandes R.L., Integrability and reduction of Hamiltonian actions on Dirac manifolds, arXiv:1311.7398.

[4] Bursztyn H., On gauge transformations of Poisson structures, in Quantum Field Theory and Noncommutative Geometry, Lecture Notes in Phys., Vol. 662, Springer, Berlin, 2005, 89-112.

[5] Bursztyn H., Radko O., Gauge equivalence of Dirac structures and symplectic groupoids, Ann. Inst. Fourier (Grenoble) $\mathbf{5 3}$ (2003), 309-337, math.SG/0202099.

[6] Chavel I., Riemannian geometry. A modern introduction, Cambridge Studies in Advanced Mathematics, Vol. 98, 2nd ed., Cambridge University Press, Cambridge, 2006.

[7] Courant T.J., Dirac manifolds, Trans. Amer. Math. Soc. 319 (1990), 631-661.

[8] Courant T.J., Weinstein A., Beyond Poisson structures, in Action Hamiltoniennes de Groupes. Troisième Théorème de Lie (Lyon, 1986), Travaux en Cours, Vol. 27, Hermann, Paris, 1988, 39-49.

[9] Crainic M., Mărcuț I., A normal form theorem around symplectic leaves, J. Differential Geom. 92 (2012), 417-461, arXiv:1009.2090.

[10] Davis B.L., Wade A., Dirac structures and gauge symmetries of phase spaces, Rend. Semin. Mat. Univ. Politec. Torino 67 (2009), 123-135.

[11] Dufour J.-P., Wade A., On the local structure of Dirac manifolds, Compos. Math. 144 (2008), 774-786, math.SG/0405257.

[12] Frejlich P., Mărcuţ I., Poisson transversals I. The normal form theorem, arXiv:1306.6055.

[13] Jotz M., Ratiu T.S., Induced Dirac structures on isotropy-type manifolds, Transform. Groups 16 (2011), 175-191, arXiv:1008.2280.

[14] Jotz M., Ratiu T.S., Śniatycki J., Singular reduction of Dirac structures, Trans. Amer. Math. Soc. 363 (2011), 2967-3013, arXiv:0901.3062.

[15] Koláŕ I., Michor P.W., Slovák J., Natural operations in differential geometry, Springer-Verlag, Berlin, 1993.

[16] Marsden J., Montgomery R., Ratiu T., Reduction, symmetry, and phases in mechanics, Mem. Amer. Math. Soc. 88 (1990), iv+110 pages. 
[17] Miranda E., Zung N.T., A note on equivariant normal forms of Poisson structures, Math. Res. Lett. 13 (2006), 1001-1012, math.SG/0510523.

[18] Ševera P., Weinstein A., Poisson geometry with a 3-form background, Progr. Theoret. Phys. Suppl. 144 (2001), 145-154, math.SG/0107133.

[19] Śniatycki J., Differential geometry of singular spaces and reduction of symmetry, New Mathematical Monographs, Vol. 23, Cambridge University Press, Cambridge, 2013.

[20] Stefan P., Accessible sets, orbits, and foliations with singularities, Proc. London Math. Soc. 29 (1974), 699-713.

[21] Sussmann H.J., Orbits of families of vector fields and integrability of distributions, Trans. Amer. Math. Soc. 180 (1973), 171-188.

[22] Vaisman I., Lectures on the geometry of Poisson manifolds, Progress in Mathematics, Vol. 118, Birkhäuser Verlag, Basel, 1994.

[23] Vaisman I., Coupling Poisson and Jacobi structures on foliated manifolds, Int. J. Geom. Methods Mod. Phys. 1 (2004), 607-637, math.SG/0402361.

[24] Vaisman I., Foliation-coupling Dirac structures, J. Geom. Phys. 56 (2006), 917-938, math.SG/0412318.

[25] Vorobjev Yu., Coupling tensors and Poisson geometry near a single symplectic leaf, in Lie Algebroids and Related Topics in Differential Geometry (Warsaw, 2000), Banach Center Publ., Vol. 54, Polish Acad. Sci. Inst. Math., Warsaw, 2001, 249-274, math.SG/0008162.

[26] Vorobjev Yu., Poisson equivalence over a symplectic leaf, in Quantum algebras and Poisson geometry in mathematical physics, Amer. Math. Soc. Transl. Ser. 2, Vol. 216, Amer. Math. Soc., Providence, RI, 2005, 241-277, math.SG/0503628.

[27] Vorobiev Yu., Averaging of Poisson structures, in Geometric Methods in Physics, AIP Conf. Proc., Vol. 1079, Amer. Inst. Phys., Melville, NY, 2008, 235-240.

[28] Vorobiev Yu., The averaging in Hamiltonian systems on slow-fast phase spaces with $\mathbb{S}^{1}$ symmetry, Phys. Atomic Nuclei 74 (2011), 1770-1774.

[29] Wade A., Poisson fiber bundles and coupling Dirac structures, Ann. Global Anal. Geom. 33 (2008), 207-217, math.SG/0507594. 